\title{
Performance evaluation study of strategic nuclear deterrent
}

\author{
Tiejian YANG ${ }^{1}$, Minle WANG ${ }^{1}$, Song TIAN ${ }^{1,2}$, Baoshun ZHOU $^{3}$, Lan $^{4}{ }^{4}$ \\ ${ }^{1} \mathrm{Xi}$ 'an Hi-tech institute, Xi'an Shaanxi 710025, China \\ ${ }^{2}$ Chongqing communication college, Chongqing 400035, China \\ ${ }^{3}$ Troops No.96630, Beijing 102206, China \\ ${ }^{4}$ Hospital 150, Luoyang 471000, China
}

\begin{abstract}
To build a nuclear deterrent effectiveness evaluation model. Essentially based on nuclear deterrence, nuclear deterrence factors are analyzed and strategic nuclear deterrent performance system model is built. Based on Agent, nuclear deterrence performance assessment analysis model is constructed, the model is practical to solving complex problems and emerging issues performance assessment studies of nuclear deterrence has a strong reference value.
\end{abstract}

Key words: nuclear deterrent; performance evaluation; strategy

\section{Introduction}

The operation of the process of strategic nuclear deterrence, weapons of deterrence have the military strength and the deter- mination of the use of strength is the implementation deterrence premise ${ }^{[1]}$. Deterrence has been effective in the transmission of information and the key to implementation of deterrence, the message can be expressed as a conditional threat ${ }^{[2-}$ 3] (see figure 1): "If you take action Y, I will take action $\mathrm{X}$ to punish you, and your loss is greater than you gains. "The above discussion reflects the cost of inputs-benefit analysis of thinking, the thinking behind the logic: deterrence conditions to be established, the "deterrence through deterrence performance assessment (challenger on the War risk assessment and cost assessment): deterrence squarecounter the challenges caused by the party's loss is greater than the revenue expected offensive; challenger such psychological effects: L (loss) $-\mathrm{G}$ (benefit) $>0$ ", the loss of challenger attack courage to give up the threat ${ }^{[4-5]}$.
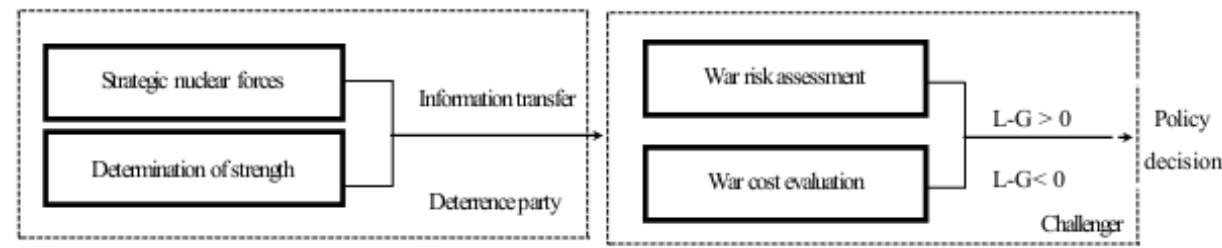

Figure 1: Strategic nuclear deterrence functioning

2. Performance architecture of strategic nuclear deterrent

\subsection{Principles of Architecture}

The set $S$ that the deterrence square strategic nuclear deterrence performance sys- 
tem is composed of different system components:

$$
\mathrm{S}=\left\{\mathrm{s}_{1_{\mathrm{s}}} s_{2} \ldots \ldots s_{n}\right\} \text {, each } \mathrm{s}_{i} \text { have }
$$

$\mathrm{m}_{i}$ factors, system is composed of the

total number of systems $\mathrm{m}$ :

$$
m=\sum_{i=1}^{n} m_{i}
$$

Each type of system performance indicators:

$$
\overline{P_{z}}=\left\{p_{i, r_{1}} p_{i, r_{2}} \ldots \ldots p_{i, r_{n}}\right\}
$$

Each $\bar{P}_{\imath}$ has $\mathrm{r}_{i} \operatorname{dim}$ :

$$
\mathrm{r}=\sum_{i=1}^{n} r_{i}
$$

\subsection{Performance system model of stra- tegic nuclear deterrent}

Nuclear forces refers to the number of missiles, penetration performance, viability assured destruction.

(1) Use of the verification force determination system. Is the attitude and policy of a State to use nuclear power.

(2) Nuclear combat capability of the system. Refers to the scheme for the application of nuclear forces and the daily nuclear forces combat training effect.

(3) Nuclear damage mental endurance system. Various countries because of the different culture, way of life, the psychological ability to have suffered nuclear strikes are not the same.

Based on the above analysis, this paper presents a structural model of nuclear deterrence, performance related factors, as shown in figure 2.

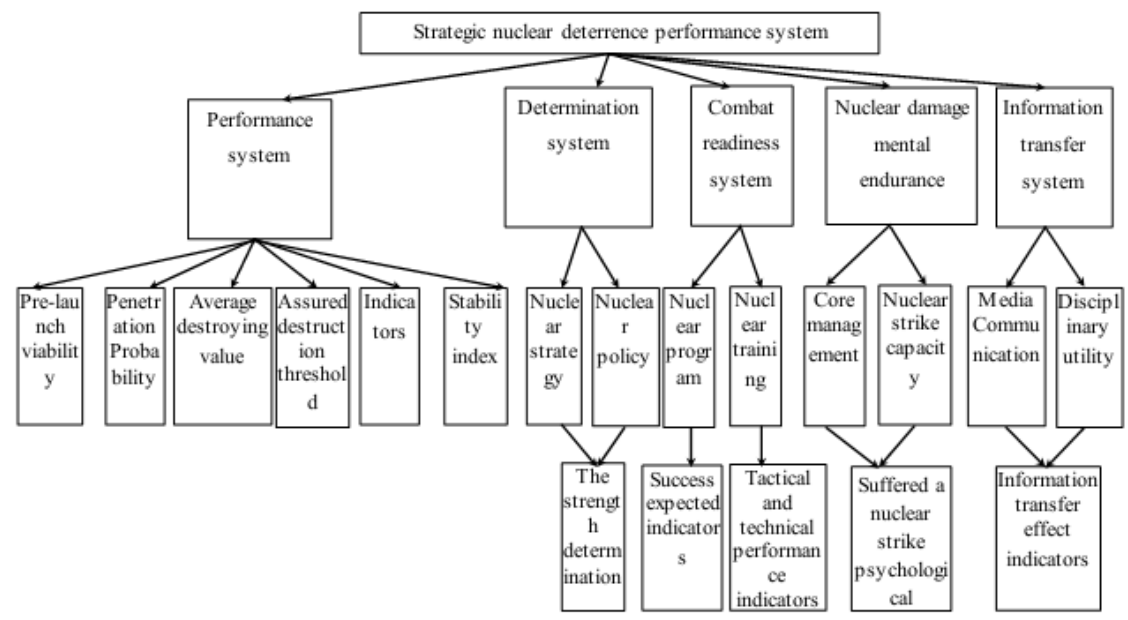

Figure 2: Effectiveness of nuclear deterrence model the structure of the relevant factors

\subsection{Effectiveness evaluation model of strategic nuclear deterrent}

In order to describe the performance assessment of the strategic nuclear deterrent, Agent analysis modeling-based approach to the establishment of the strategic nuclear deterrent effectiveness evaluation model as shown in the figure 3 .
The realization of the strategic nuclear deterrent effectiveness evaluation model, mainly achieved by assessing the Agent assess the Agent to have the database, information gathering, deterrence feedback quantizer, the ability to self-learning, through deterrence feedback quantizer, and deterrence degree standard library, to 
achieve performance assessment.

Deterrence degree of recognition quantify the results of the assessment, product assessment is based primarily on the effect and impact of each strategic deterrence factors. For comparison, according to the results of deterrence degree standard library corresponding warning is given in accordance with the level exceeds the critical point. The deterrence degree standard library to establish, as given in Table 1 deterrence evaluation matrix, the table can be given the deterrence size deterrence size hierarchy can also be given in the number.

Correspondence between deterrence level with the deterrent effect: highly efficient deterrent 1 to 3 ; and efficient deterrence 4 to 8 ; in effective deterrence 9 to 13, low deterrence 14 to 20 deterrence; deterrent effect can be ignored: more than 20. Under different conditions of the deterrent level meaning given a different design. The Agent received the results of analysis and evaluation, and optimized their work, so that the strategic nuclear deterrent effect has been comprehensively improved.

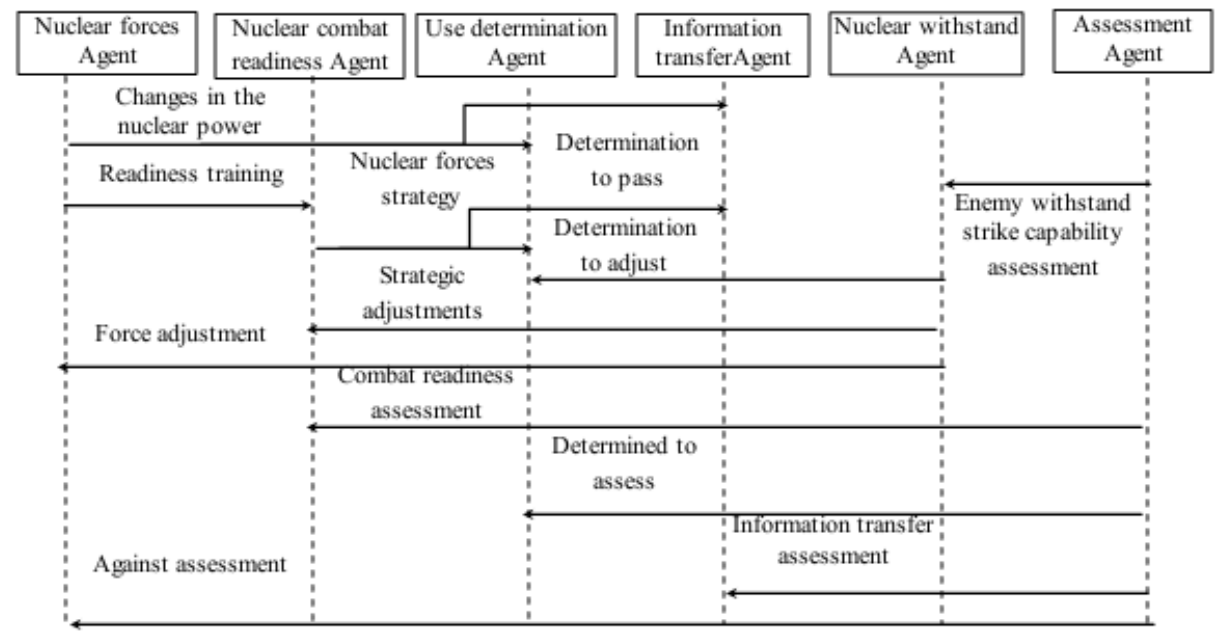

Figure 3: Effectiveness evaluation model of strategic nuclear deterrent

Table 1 Deterrence evaluation matrix

\begin{tabular}{|c|c|c|c|c|c|c|}
\hline \multicolumn{2}{|c|}{} & \multicolumn{5}{|c|}{ effect } \\
\cline { 3 - 7 } deterrence degrees & very good & good & general & poor & very poor \\
\cline { 3 - 7 } victory & I & $\begin{array}{c}1 \text { extremely } \\
\text { efficient }\end{array}$ & 2 extremely efficient & 6 efficient & 8 Efficient & 12 In effect \\
\hline key & II & $\begin{array}{c}3 \text { extremely } \\
\text { efficient }\end{array}$ & 4 efficient & 7 Efficient & 11 In effect & 15 in effect \\
\hline moderate & III & 5 efficient & 9 in effect & 10 in effect & 14 in effect & 16 in effect \\
\hline neglect & IV & 13 In effect & 17 Inefficient & 18 in effect & 19 in effect & 20 in effect \\
\hline
\end{tabular}




\section{Conclusions}

Assessment of the effectiveness of nuclear deterrence is a complex problem involving many factors, the study found that the main factors and secondary factors, and potential factors, in order to make a comprehensive analysis of the effectiveness of nuclear deterrence, we provide the necessary support for the use of nuclear strategy. Agent model with self-learning ability, diversity and resolve performance assessment emerged with a strong practical, can provide support for the development of nuclear strategy and weapons development.

\section{References}

[1]http://www.dtic.mil/doarine/jel/new_p ubs/jpl_02.pdf.

[2] Henry kissinger. The Necessity for Choice. Garden city. New York: Doubleday, 1962, p.12.

[3] James R.Schlesinger. International Security (Summer 1985). 10(1):3-12.

[4] Elain M.Crossman.Top U.S. General Spurns Obama Pledge to Reduce Nuclear Alert Posture, Global Security Newswire, Feb. 272009.

[5] G lenn H. Snyder, D eterrence and Defense (Press: Princeton University Press, 1961) 